International Conference Mathematical and Computational Biology 2011

International Journal of Modern Physics: Conference Series

Vol. 9 (2012) 44-51

C World Scientific Publishing Company

DOI: $10.1142 / \mathrm{S} 2010194512005089$

\title{
SOLVING COCOA POD SIGMOID GROWTH MODEL WITH NEWTON RAPHSON METHOD
}

\author{
ALBERT LING SHENG CHANG \\ Malaysian Cocoa Board, \\ $5^{\text {th }} \& 6^{\text {th }}$ Floor, Wisma SEDCO, Locked Bag 211, \\ 88999 Kota Kinabalu, Sabah \\ albert@koko.gov.my \\ NAVIES MAISIN \\ Cocoa Research \& Development Centre, Mile 10, \\ Apas Road, P. O. Box 60237, 91012 Tawau \\ navie@koko.gov.my
}

\begin{abstract}
Cocoa pod growth modelling are useful in crop management, pest and disease management and yield forecasting. Recently, the Beta Growth Function has been used to determine the pod growth model due to its unique for the plant organ growth which is zero growth rate at both the start and end of a precisely defined growth period. Specific pod size $(7 \mathrm{~cm}$ to $10 \mathrm{~cm}$ in length) is useful in cocoa pod borer (CPB) management for pod sleeving or pesticide spraying. The Beta Growth Function is well-fitted to the pods growth data of four different cocoa clones under non-linear function with time $(t)$ as its independent variable which measured pod length and diameter weekly started at 8 weeks after fertilization occur until pods ripen. However, the same pod length among the clones did not indicate the same pod age since the morphological characteristics for cocoa pods vary among the clones. Depending on pod size for all the clones as guideline in CPB management did not give information on pod age, therefore it is important to study the pod age at specific pod sizes on different clones. Hence, Newton Raphson method is used to solve the nonlinear equation of the Beta Growth Function of four different group of cocoa pod at specific pod size.
\end{abstract}

Keywords: Cocoa; Cocoa pod growth; Beta growth function; Newton-Raphson method.

\section{Introduction}

Plant growth models have been widely used to provide useful information on plant growth process. In cocoa, mathematical models also being widely used to describe the growth process especially on pod development. Early studies related to cocoa pod development were reported by Nichols (1964) and Wood (1985). Navies (2009) developed an cocoa pod sigmoid growth model using the Beta Growth Function to determine the pod growth in terms of pod length and pod diameter for four different clones, namely COCA 3370-5, KKM22, PBC123 and BR25. The Beta Growth Function was used to determine the pod growth model due to its unique for the plant growth which is zero growth rate at both the start and end of a precisely defined growth period (Yin 
et al., 2003). It is well-fitted to the pods growth data under non-linear function with time $(t)$ as its independent variable representing the pod age from fertilization until pods ripen.

The Beta Growth Function on determine cocoa pod growth in terms of pod length and diameter by knowing the pod age from fertilization is useful in pest management work. This is important especially during the application on pod sleeving approach in managing the Cocoa Pod Borer (CPB) which required the pod with sizes of $7 \mathrm{~cm}$ to $10 \mathrm{~cm}$ covered with transparent plastic bag (Azhar, 2009). Besides that, spraying should also be commenced when the pods reach more than $7 \mathrm{~cm}$ in length. The reason for spraying pods at $7 \mathrm{~cm}$ in length due to its preference for CPB to lay eggs (Azhar and Long, 1996). This could be the pods at that stage are more nutritious or because of the presence or absence of specific chemical or morphological cues (Thompson and Pellmyr, 1991). Moreover, cocoa pod at $7 \mathrm{~cm}$ to $10 \mathrm{~cm}$ in length is a guideline for the growers to use in deciding action to manage CPB. However, the pod length couldn't indicate the same pod age because morphological characteristics of cocoa pods vary between the clones. Therefore, study on the difference on the pod age at the same pod length or diameter among the clones is essential. Applications of the cocoa pod sigmoid growth model is impeded by the analytical difficulties associated with solving it. The cocoa pod sigmoid growth model presents a non-linear Beta Growth functional equation which is known to have a unique solution at certain pod length or diameter. The solution, however, cannot be derived exactly using standard algebraic techniques. The non-linear Beta Growth functional equation can only be solved numerically using computer. In this paper, the Newton Raphson Method will be used for computing approximate solutions to the nonlinear cocoa pod sigmoid growth model. The pod age at several pod length and diameter will be estimated by solving the non-linear Beta Growth equation on four different clones.

\section{Model Derivation}

The life cycle of a plant can be divided into three sub-phase, namely an early accelerating phase, a linear phase and a saturation phase for ripening (Goudriaan and Van Laar, 1994). These phases normally can be explained mathematically such as the growth pattern follows a sigmoid curve, and the growth rate follows a bell-shaped curve. The sigmoid pattern normally can be represented using a curve linear equation which gives a gradual transition from one phase to the next. There were few mathematical models being derived to represent the growth function. Goudriaan (1994) derived the expolinear equation with two smooth transitions to describe the pattern of leaf area index as given follows;

$$
w=\frac{c_{m}}{r_{m}} \ln \left[\frac{1+e^{r_{m}\left(t-t_{0}\right)}}{1+e^{r_{m}\left(t-t_{0}-w_{\max } / c_{m}\right)}}\right]
$$

where $w$ is mass, $t$ is time, $t_{0}$ is the moment at which the linear phase effectively begins, and $c_{m}$ and $r_{m}$ are maximum growth rate in the linear phase and maximum relative growth rate (RGR) in th exponential phase, respectively. 
However, the Eq. (1) gives a symmetrical sigmoid pattern around time $t_{0}+w_{\max } /\left(2 c_{m}\right)$. This might not work well in explaining the plant growth as most of the plant growth work in asymmetrical function. An alternative way to generate an asymmetrical growth curve is to use the Gompertz function (Yin et al., 2003) which need only three parameters as given follows;

$$
w=w_{\max } e^{-e^{-k\left(t-t_{m}\right)}}
$$

where time , $t_{m}$ will give the absolute growth rate the maximum at $w_{\max } / e$ and the RGR is equal to $k$.

The Eq. (1) and (2) can't predict the actual weight equal to $w_{\max }$ because they all have the line $w=w_{\max }$ as their upper asymptote when time tends to infinity. This is contradicting the pattern of growth as growth period is infinite in those equations. A segmented terminate function is needed to find a final weight at the end of a precisely defined growth period. Yin et al. (2003) has derived a new growth function known as a flexible asymmetrical sigmoid growth function or the Beta Growth Function as follows;

$$
w=w_{\max }\left(1+\frac{t_{e}-t}{t_{e}-t_{m}}\right)\left(\frac{t}{t_{e}}\right)^{\frac{t_{e}}{t_{e}-t_{m}}} \quad \text { with } 0 \leq t_{\mathrm{m}} \leq t_{\mathrm{e}}
$$

where $w_{\max }$ is the maximum value of $w$ when reaching at time $t_{\mathrm{e}}$ and $t_{m}$ is the time at maximum growth rate.

\subsection{Cocoa pod sigmoid growth model}

Navies (2009) fitted the cocoa pod length and diameter that were measured from fertilization to ripen into the Eq. (3). The data was collected weekly from September to December 2005 at Cocoa Research and Development Center Tawau, Sabah. Four cocoa clones were used in his study, i.e. COCA3370-5, KKM22, PBC123 and BR25. The fitted pod growth data to the Beta Growth Function in Eq. (3) for four cocoa clones are shown in Table 1 and 2. The Beta Growth Function fitted well to the data as the R square obtained were above $95 \%$.

Table 1. The cocoa pod sigmoid growth model on the pod length of four cocoa clones.

\begin{tabular}{ccc}
\hline Clones & Growth function of pod length $(\mathrm{cm})^{\mathrm{a}}$ & $\mathrm{R}^{2}$ \\
\hline COCA 3370-5 & $w=13.814\left(1+\frac{136.591-t}{104.331}\right)\left(\frac{t}{136.591}\right)^{1.309}$ & 0.982 \\
KKM22 & $w=12.768\left(1+\frac{113.914-t}{110.289}\right)\left(\frac{t}{113.914}\right)^{1.033}$ & 0.976 \\
PBC123 & $w=14.583\left(1+\frac{116.946-t}{109.028}\right)\left(\frac{t}{116.946}\right)^{1.073}$ & 0.995 \\
BR25 & $w=14.719\left(1+\frac{114.005-t}{103.590}\right)\left(\frac{t}{114.005}\right)^{1.101}$ & 0.977 \\
\hline
\end{tabular}

Note: $t$ is measured as days from fertilization

${ }^{\mathrm{a}}$ Cited from Navies (2009). 
Table 2. The cocoa pod sigmoid growth model on the pod diameter of four cocoa clones.

\begin{tabular}{ccc}
\hline Clones & Growth function of pod length $(\mathrm{cm})^{\mathrm{a}}$ & $\mathrm{R}^{2}$ \\
\hline COCA 3370-5 & $w=7.434\left(1+\frac{148.714-t}{89.477}\right)\left(\frac{t}{148.714}\right)^{1.662}$ & 0.990 \\
KKM22 & $w=7.673\left(1+\frac{124.520-t}{76.818}\right)\left(\frac{t}{124.520}\right)^{1.621}$ & 0.959 \\
PBC123 & $w=6.955\left(1+\frac{129.917-t}{91.508}\right)\left(\frac{t}{129.917}\right)^{1.420}$ & 0.973 \\
BR25 & $w=7.434\left(1+\frac{119.069-t}{71.607}\right)\left(\frac{t}{119.069}\right)^{1.663}$ & \\
\hline $\begin{array}{l}\text { Note: } t \text { is measured as days from fertilization } \\
\text { a Cited from Navies (2009). }\end{array}$
\end{tabular}

The cocoa pod sigmoid growth model estimated the maximum pod length for each clone in the range of $12.77 \mathrm{~cm}$ to $14.72 \mathrm{~cm}$ (Table 1 ). The maximum pod diameter for each clone was ranged from $6.96 \mathrm{~cm}$ to $7.67 \mathrm{~cm}$ (Table 2). In addition, the cocoa pod sigmoid growth model also showed that the time (days) to reach the maximum growth rates and values varied among the clones. This is due to the genotype factor which influenced the most on the morphological characteristics of cocoa pod. In order to determine the pod age among the clones at the same pod length or diameter, the cocoa pod sigmoid growth model need to be solved inversely.

However, the non-linear Beta Growth functional equation at certain pod length and diameter cannot be derived exactly using standard algebraic techniques. The Newton Raphson Method will be used to compute approximate solutions to the non-linear equations at several pod length and diameter on four different clones.

\section{Newton Raphson Method}

The Newton Raphson method is known as open method which requires initial guess of the root of a non-linear equations $f(t)=0$ at $t_{\mathrm{i}}$, then if one draws the tangent to the curve at $f\left(t_{\mathrm{i}}\right)$, the point $t_{\mathrm{i}+1}$ where the tangent crosses the $\mathrm{x}$-axis is an improved estimate of the root. The definition of the slope of a function at $t=t_{\mathrm{i}}$ will derive the Newton Raphson formula as follows;

$$
\begin{aligned}
f^{\prime}\left(t_{\mathrm{i}}\right) & =\tan \theta \\
& =\frac{f\left(t_{i}\right)-0}{t_{i}-t_{i+1}} \\
t_{i+1} & =t_{i}-\frac{f\left(t_{i}\right)}{f\left(t_{i}\right)}
\end{aligned}
$$

where $f^{\prime}\left(t_{\mathrm{i}}\right)$ is the first derivative of the function $f^{\prime}\left(t_{\mathrm{i}}\right)$. 


\subsection{The Netwon Raphson algorithm}

There are four steps involved in Netwon Raphson algorithm to find the root of an equation $f(t)=0$ as follows;

Step 1: Evaluate $f^{\prime}\left(t_{\mathrm{i}}\right)$ symbolically

Step 2: Use an initial guess of the root, $t_{\mathrm{i}}$ to estimate the new value of the root, $t_{\mathrm{i}+1}$, as given in Eq. (4).

Step 3: Find the absolute relative approximate error $\left|\epsilon_{a}\right|=\left|\frac{t_{i+1}-t_{i}}{t_{i+1}}\right| \times 100$.

Step 4: Compare the absolute relative approximate error with the pre-specified relative error tolerance, $\epsilon_{s}$ which was set at default of 0.005 . If $\left|\epsilon_{a}\right|>\epsilon_{s}$, then go to Step 2, else stop the algorithm. The maximum number of iterations allowed in this algorithm will be 100 before the algorithm is terminated.

\subsection{Solving the cocoa pod sigmoid growth model}

In the cocoa pod sigmoid growth model, we will estimate the pod age at pod length of $7 \mathrm{~cm}$ till $13 \mathrm{~cm}$ and pod diameter of $1 \mathrm{~cm}$ till $7 \mathrm{~cm}$. The range was chosen based on the susceptible pods to CPB attacked. The derivation on the non-linear Beta Growth functional equation at pod length, $l$ and diameter, $d$ are given as follows;

$$
\begin{gathered}
f\left(t_{i}\right)=w_{\max }\left(1+\frac{t_{e}-t}{t_{e}-t_{m}}\right)\left(\frac{t}{t_{e}}\right)^{\frac{t_{e}}{t_{e}-t_{m}}}-l \quad \text { where } 7 \leq l \leq 13 \\
f\left(t_{i}\right)=w_{\max }\left(1+\frac{t_{e}-t}{t_{e}-t_{m}}\right)\left(\frac{t}{t_{e}}\right)^{\frac{t_{e}}{t_{e}-t_{m}}}-d \quad \text { where } 1 \leq d \leq 7
\end{gathered}
$$

In step 1, the first order derivative on the Eq. (5) or (6) is given as follows;

$f^{\prime}\left(t_{i}\right)=\left(-\frac{w_{\max }}{t_{e}-t_{m}}\right)\left(\frac{t}{t_{e}}\right)^{\frac{t_{e}}{t_{e}-t_{m}}}+\left(w_{\max }+\left(\frac{w_{\max } \times t_{e}}{t_{e}-t_{m}}\right)-\left(\frac{w_{\max } \times t}{t_{e}-t_{m}}\right)\right)\left(\frac{1}{t_{e}}\right)^{\frac{t_{e}}{t_{e}-t_{m}}}\left(\frac{t_{e}}{t_{e}-t_{m}}\right) \times t^{\left(\frac{t_{e}}{t_{e}-t_{m}}-1\right)}$

For example, the first order derivative function, $f^{\prime}\left(t_{\mathrm{i}}\right)$ at pod length $7 \mathrm{~cm}$ for clone COCA $3370-5$ is given as follows;

$f^{\prime}\left(t_{i}\right)=\left(-\frac{13.81}{104.33}\right)\left(\frac{t}{13.81}\right)^{1.31}+\left(13.81+\left(\frac{1886.87}{104.33}\right)-\left(\frac{13.81 \times t}{104.33}\right)\right)\left(\frac{1}{136.59}\right)^{1.31}(1.31) \times t^{(0.31)}$

In Step 2, we start an initial guess of the root, $t_{\mathrm{i}}$ at 25 to estimate the new value of the root, $t_{\mathrm{i}+1}$, using the Eq. (4) which gave the following calculations;

$$
\begin{aligned}
t_{i+1} & =25-\frac{f(25)}{f(25)} \\
& =51.38 \text { days from fertilization }
\end{aligned}
$$

In the step 3 and 4 , calculation on the absolute relative approximate error $\left|\epsilon_{a}\right|$ at the end of iteration 1 is

$$
\begin{aligned}
\left|\epsilon_{a}\right| & =\left|\frac{51.38-25}{51.38}\right| \times 100 \\
& =51.34 \%
\end{aligned}
$$


As the absolute relative approximate error $\left|\epsilon_{a}\right|>\epsilon_{s}$ which is set at 0.005 or $0.5 \%$. Then, the second iteration will be continued until the absolute relative approximate error $\left|\epsilon_{a}\right|<$ 0.005 or $0.5 \%$ as given in Table 3 .

Table 3. Number of iteration needed in solving the cocoa pod sigmoid growth.

\begin{tabular}{cccc}
\hline Iteration number & $\mathbf{t}_{\mathbf{i}}$ & $\mathbf{f}\left(\mathbf{t}_{\mathbf{i}}\right)$ & $\left|\in_{a}\right| \%$ \\
\hline 1 & 25 & -3.9048 & 51.3429 \\
2 & 51 & -0.0160 & 0.2203 \\
3 & 52 & 0.0000 & 0.0001 \\
4 & 52 & 0.0000 & 0.0000 \\
\hline
\end{tabular}

The Table 3 showed the Newton Raphson method converged to value 52 after 3 iterations where the absolute relative approximate error $\left|\epsilon_{a}\right|<0.005$. The similar algorithm is also applied to other ranges of pod length and diameter of other clones.

\section{Results}

The Newton Raphson method converged very fast within 3 to 5 iterations except for pod diameter at $7 \mathrm{~cm}$ which converged after 7 iterations to achieve the absolute relative approximate error $\left|\epsilon_{a}\right|<0.005$ (Table 4 and 5).

Table 4. Pod age estimation of four different clones at certain pod length using Newton Raphson method.

\begin{tabular}{lcccc}
\hline Clone & Pod Length (cm) & Pod Age (Days) & No. Iterations & $\left|\in_{\boldsymbol{a}}\right|$ \\
\hline BR25 & 7.0 & 35 & 4 & 0.0000 \\
PBC123 & 7.0 & 35 & 4 & 0.0000 \\
KKM22 & 7.0 & 38 & 4 & 0.0000 \\
COCA3370-5 & 7.0 & 52 & 3 & 0.0001 \\
\hline BR25 & 8.0 & 40 & 4 & 0.0000 \\
PBC123 & 8.0 & 41 & 4 & 0.0000 \\
KKM22 & 8.0 & 45 & 4 & 0.0001 \\
COCA3370-5 & 8.0 & 59 & 4 & 0.0024 \\
\hline BR25 & 9.0 & 46 & 4 & 0.0000 \\
PBC123 & 9.0 & 47 & 3 & 0.0001 \\
KKM22 & 9.0 & 53 & 4 & 0.0006 \\
COCA3370-5 & 9.0 & 66 & 4 & 0.0005 \\
\hline BR25 & 10.0 & 53 & 4 & 0.0002 \\
PBC123 & 10.0 & 54 & 3 & 0.0001 \\
KKM22 & 10.0 & 62 & 5 & 0.0000 \\
COCA3370-5 & 10.0 & 75 & 4 & 0.0007 \\
\hline BR25 & 11.0 & 60 & 4 & 0.0003 \\
PBC123 & 11.0 & 61 & 4 & 0.0000 \\
KKM22 & 11.0 & 72 & 5 & 0.0000 \\
COCA3370-5 & 11.0 & 84 & 4 & 0.0002 \\
\hline BR25 & 12.0 & 68 & 4 & 0.0029 \\
PBC123 & 12.0 & 70 & 4 & 0.0001 \\
KKM22 & 12.0 & 87 & 5 & 0.0030 \\
COCA3370-5 & 12.0 & 95 & 4 & 0.0034 \\
\hline BR25 & 13.0 & 77 & 5 & 0.0000 \\
PBC123 & 13.0 & 80 & 4 & 0.0033 \\
COCA3370-5 & 13.0 & 109 & 5 & 0.0001 \\
\hline & & & &
\end{tabular}


The results indicate that at the specific pod size (length and diameter), pod age were varied among the clones (Table 4 and 5). This indicates pod growth rate varies among the clones. Higher pod growth rate will take quick time (days) to achieve specific pod size. It was clear through this study that BR25 have the highest pod growth rate, while COCA3370-5 was on the other side. For example, the pod age for clone BR25 was 35 days from fertilization while COCA3370-5 was 52 days from fertilization at $7 \mathrm{~cm}$ in pod length. The range of pod age was 40 to 59 days from fertilization for $8 \mathrm{~cm}$ in pod length; 46 to 66 days from fertilization for $9 \mathrm{~cm}$ in pod length; 53 to 75 days from fertilization for $10 \mathrm{~cm}$ in pod length; 60 to 84 days from fertilization for $11 \mathrm{~cm}$ in pod length; 68 to 95 days from fertilization for $12 \mathrm{~cm}$ in pod length; and 77 to 109 days from fertilization for $13 \mathrm{~cm}$ in pod length.

On diameter, the range of pod age was 19 to 26 days from fertilization for $1 \mathrm{~cm}$ in pod diameter; 32 to 42 days from fertilization for $2 \mathrm{~cm}$ in pod diameter; 45 to 56 days from fertilization for $3 \mathrm{~cm}$ in pod diameter; 45 to 56 days from fertilization for $4 \mathrm{~cm}$ in pod diameter; 56 to 70 days from fertilization for $5 \mathrm{~cm}$ in pod diameter; 81 to 101 days from fertilization for $6 \mathrm{~cm}$ in pod diameter and 98 to 124 days from fertilization for $7 \mathrm{~cm}$ in pod diameter.

Table 5. Pod age estimation of four different clones at certain pod diameter using Newton Raphson method.

\begin{tabular}{lcccc}
\hline Clone & Pod Diameter $(\mathbf{c m})$ & Pod Age (Days) & No. Iterations & $\left|\in_{\boldsymbol{a}}\right|$ \\
\hline PBC123 & 1.0 & 19 & 3 & 0.0000 \\
BR25 & 1.0 & 21 & 3 & 0.0000 \\
KKM22 & 1.0 & 21 & 3 & 0.0000 \\
COCA3370-5 & 1.0 & 26 & 4 & 0.0002 \\
\hline PBC123 & 2.0 & 32 & 3 & 0.0016 \\
BR25 & 2.0 & 34 & 4 & 0.0000 \\
KKM22 & 2.0 & 34 & 4 & 0.0000 \\
COCA3370-5 & 2.0 & 42 & 4 & 0.0005 \\
\hline BR25 & 3.0 & 45 & 4 & 0.0000 \\
KKM22 & 3.0 & 45 & 3 & 0.0012 \\
PBC123 & 3.0 & 45 & 4 & 0.0000 \\
COCA3370-5 & 3.0 & 56 & 5 & 0.0000 \\
\hline BR25 & 4.0 & 56 & 4 & 0.0001 \\
KKM22 & 4.0 & 56 & 4 & 0.0001 \\
PBC123 & 4.0 & 59 & 4 & 0.0000 \\
COCA3370-5 & 4.0 & 70 & 5 & 0.0020 \\
\hline BR25 & 5.0 & 68 & 5 & 0.0038 \\
KKM22 & 5.0 & 68 & 4 & 0.0019 \\
PBC123 & 5.0 & 73 & 4 & 0.0000 \\
COCA3370-5 & 5.0 & 85 & 5 & 0.0000 \\
\hline BR25 & 6.0 & 81 & 5 & 0.0000 \\
KKM22 & 6.0 & 81 & 5 & 0.0000 \\
PBC123 & 6.0 & 91 & 4 & 0.0000 \\
COCA3370-5 & 6.0 & 101 & 5 & 0.0022 \\
\hline KKM22 & 7.0 & 98 & 5 & 0.0000 \\
BR25 & 7.0 & 99 & 5 & 0.0001 \\
COCA3370-5 & 7.0 & 124 & 7 & 0.0000 \\
\hline & & & &
\end{tabular}




\section{Conclusions}

The solutions computed numerically from the non-linear Beta Growth Functional equation using Newton Raphson method suggest that the cocoa pod age of different cocoa clones at which CPB would preferred to infest by knowing the pod length and diameter. This findings could be included in CPB management programme for better control of the pest. In addition, the growers could used the information on pod ages to determine the right time in harvesting the pods.

\section{Acknowledgements}

We would like to acknowledge financial assistance provided by Sciencefund grant under Ministry of Agriculture (MOA) entitled "Developing an user-friendly interactive decision tool for CPB management". Besides that, I would like to thank the Director General of Malaysian Cocoa Board for allowing me to present this paper and also the Biology Director and other staffs who may contribute directly or indirectly to this paper.

\section{References}

1. X. Yin, J. Goudriaan, E.A. Lantinga, J. Vos and H.J. Spiertz. A flexible sigmoid function of determinate growth. Annals of Botany 91, 361 (2003).

2. G.A.R. Wood, From harvest to store, in Cocoa Fourth edition, Tropical Agriculture Series. ed. G.A.R. Woods and R.A. Lass. (Longman Group Ltd, Singapore, 1985), p. 444.

3. R. Nichols, Studies of fruit development of cacao (Theobroma cacao) in relation to cherelle wilt. I. Development of the pericarp. Annals of Botany 28, 619 (1964).

4. G.E. Long, and I. Azhar, The ecology of cocoa pod borer in Malaysia: Dispersion pattern for eggs among trees. MARDI Res. J. 24, 163 (1996).

5. M. Navies, Cocoa pod sigmoid growth model using 'The Beta Growth Function', in Proc. $5^{\text {th }}$ Malaysia International Cocoa Conference, 2009.

6. I. Azhar, Management of insect pests, in Cocoa Planting Manual, ed. I. Azhar, et al. (Kota Kinabalu, Sabah, 2009), p.88.

7. J.N. Thompson and O. Pellmyr, Evolution of oviposition behavior and host preference in Lepidoptera. Annual Review of Entomology 36, 65 (1991).

8. J. Goudriaan and H.H. Van Laar. Modelling potential crop growth processes. Kluwer Academic Publihers. Dordrecht, 1994.

9. J. Goudriaan. Using the expolinear growth equation to analyse resource capture, in Resource capture by crops, ed. J.L. Monteith, R.K. Scott and M.H. Unsworth (Nottigham University Press, Nottingham, 1994), p. 99. 